\title{
SPATIAL DISTRIBUTION AND COLOR OF DUST IN HALLEY'S INNER COMA
}

\author{
J. CLAIREMIDI, E. BRANDON, P. ROUSSELOT, G. MOREELS \\ Observatoire de Besançon \\ $B P 1615$ \\ 25010 BESANCON Cedex \\ FRANCE
}

\begin{abstract}
Composite images of the intensity of solar radiation scattered by dust in Halley's coma are constructed by using the three-channel spectra obtained during the approach phase of the Vega 2 spacecraft. They cover a sector centered on the nucleus that has a radius of $40000 \mathrm{~km}$ and an angular extent of $50^{\circ}$. A radial plot of dust-scattered intensity shows that it varies as the inverse of impact parameter $p$ where $p$ is smaller than $3200 \mathrm{~km}$ or higher than $7000 \mathrm{~km}$. In the intermediate $3200-7000 \mathrm{~km}$ distance range, the intensity varies as $\mathrm{p}^{-1.52}$. At longer distances, two jets are present with a contrast comparable to the gas jets which appear in the $\mathrm{OH}$ and $\mathrm{CN}$ images.

The color of dust shows a slight excess of near-UV radiation in a diffuse region between 10000 and $30000 \mathrm{~km}$ which appears to be connected with the two jets. In the region called "valley", between the jets at distances $p>25000 \mathrm{~km}$, the dust-scattered intensity shows an excess of red. The color, expressed as the ratio of intensities at 377,482 and $607 \mathrm{~nm}$ is interpreted in terms of Mie theory. It is suggested that the dust particles progressively differentiate. A proportionally more important population of small submicronic grains appears at $p>8000 \mathrm{~km}$. This population seems to correlate with the jets.
\end{abstract}

\section{Introduction}

During the observation campaign of Halley's comet, two types of instruments were used to determine the physical parameters of cometary dust: impact particle counters and optical instruments. The particle counters and analysers on board the Vega and Giotto spacecrafts provided two main results; i) the mass spectrum of dust extends to much lower masses than previously expected (Mazets et al., 1987; McDonnell et al., 1987); ii) a large proportion of grains contains a relatively high content of light elements, C, H, O and N (Jessberger et al, 1988). The optical instruments of space missions consisted of video cameras (Keller et al, 1986; Sagdeev et al, 1986), a photopolarimeter (Levasseur-Regourd et al,, 1986), an IR spectrometer (Combes et al., 1988) and a near-UV and visible spectrometer (Moreels et al., 1987). Earth and satellite-based optical instruments provided a large amount of observations in addition to the data gathered in space. Polarimetric measurements were used to precisely determine the complex index of Halley's dust (Mukai et al. 1987) and to compare cometary and interplanetary dust particles (Levasseur Regourd et al, 1990). Ground-based images in narrow-band spectral ranges, when processed with image-enhancement techniques, showed spiral structures identified as gaseous jets (A'Hearn et al., 1986; Hoban et al., 1989; Suzuki et al., 1990) .

Optical observations can be used to infer physical properties of dust grains, mainly their size. In the present study, three wavelengths : 377,482 and $607 \mathrm{~nm}$ were chosen to investigate the spatial distribution and the size range of dust. The data consist of the spectra provided by Vega 2 three-channel spectrometer on March 9, 1986 during the approach session.

A.C. Levasseur-Regourd and H. Hasegawa (eds.), Origin and Evolution of Interplanetary Dust, 277-280. (c) 1991 Kluwer Academic Publishers, Printed in Japan. 


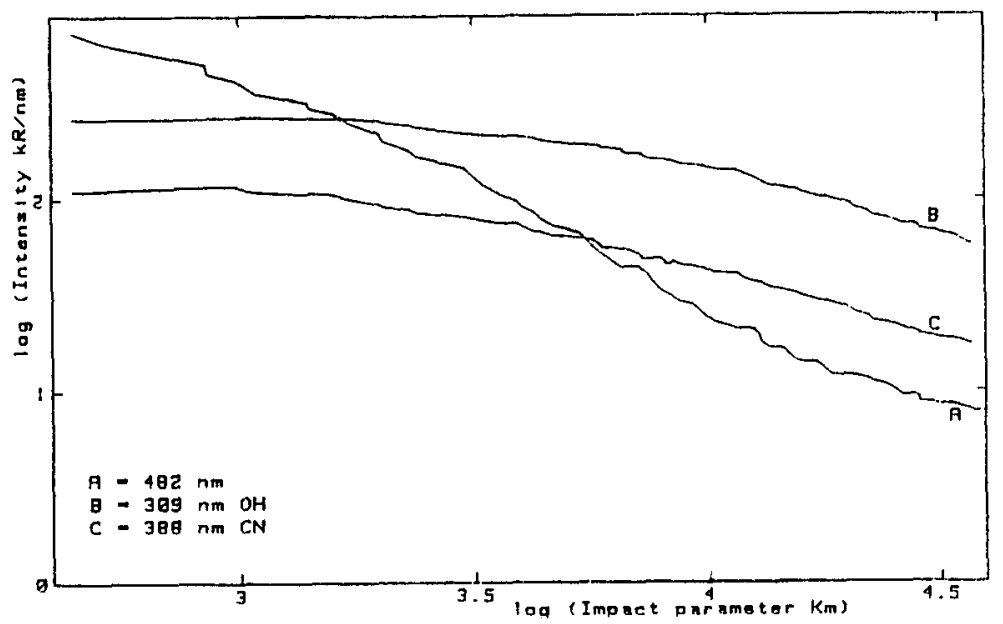

Fig.1 Radial distribution of dust-scattered intensity at $482 \mathrm{~nm}$. The log-log plot shows that the slope is -1 for $p<3200 \mathrm{~km}$ and $p>7000 \mathrm{~km}$. The slope is -1.52 in the intermediate $3200-7000 \mathrm{~km}$ range. The $\mathrm{OH}$ and $\mathrm{CN}$ intensities, corrected of the solar-scattered continuum have also been plotted

\section{Spatial distribution and color of dust in the inner coma}

A cometary spectrum presents atomic and molecular features superposed on a solar-scattered continuum which is very intense close to the nucleus. The dust-scattered component was measured at three wavelengths in the near-UV, the visual and the red parts of the spectrum. The intensity at $482 \mathrm{~nm}$ is plotted as a function of the projected distance $\mathrm{p}$ in Fig.1 with a logarithmic scale. The graph has a slope equal to -1 when $p<3200 \mathrm{~km}$ or $>7000 \mathrm{~km}$. In the intermediate $3200-7000$ $\mathrm{km}$ range, the slope is equal to -1.52 . The $\mathrm{OH}$ and $\mathrm{CN}$ intensities at 309 and $388 \mathrm{~nm}$, corrected of the dust continuum component are also plotted in Fig.1. Both molecular emissions show a typical daughter-molecule radial decrease.

The three-channel spectrometer had a scanning capacity which permits to construct composite spectral charts at selected wavelengths. The explored region is a triangle with a summit located at the nucleus. The maximum radial distance is $40000 \mathrm{~km}$. A composite image of dust intensity is presented in Fig.2. It may be compared with a similar image (Fig.3) obtained in the $\mathrm{CN}$ emission at $388 \mathrm{~nm}$ in a bandpass of $1.8 \mathrm{~nm}$ (Clairemidi et al., 1990). The spatial distributions show two differences; i) the dust intensity decreases as $1 / \mathrm{p}$ and ii) the jets appear comparatively more important in the $\mathrm{CN}$ emission. In fact, the jets are also present in dust-scattered intensity. A circular cut at $p=25000 \mathrm{~km}$ shows that the contrast at $482 \mathrm{~nm}$ between the vertical jet and the valley is equal to 3

The color of dust is represented by the intensity ratios $I_{377} / I_{482}$ (Fig.4) and $I_{607} / I_{482}$ (Fig.5). An excess of near-UV scattered light appears between 10000 and $30000 \mathrm{~km}$ mainly at the location of the jets. An excess of red intensity appears at distances $p>25000 \mathrm{~km}$ principally in the valley region. The color of dust-scattered radiation is a parameter which can provide useful information about the particle size when used in conjunction with Mie theory.

\section{Mie theory calculations. Discussion}

A Mie calculation of the scattering quantum efficiency $Q_{\text {sca }}$ was performed following the scheme given by Eaton (1984). This calculation applies to spherical particles and does not take into account the actual shape of cometary particles which are highly porous (Greenberg and Hage, 1990). However, useful information about the size should be obtained from the knowledge of wavelength where scattering is maximum. Fig.5 shows the ratios of scattering efficiencies Qsca 


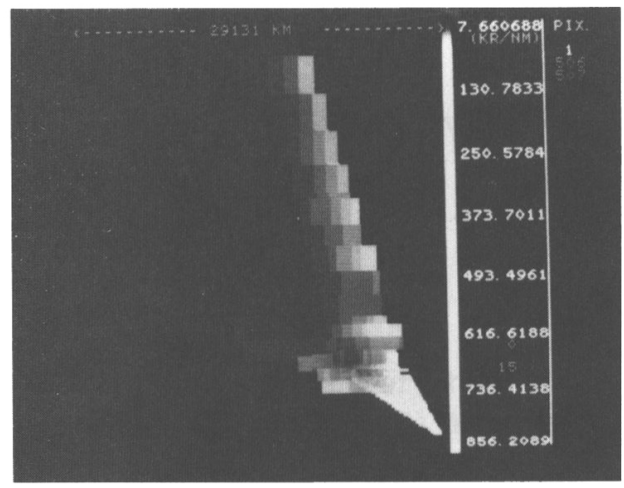

Fig. 2 Intensity of dust continuum at $482 \mathrm{~nm}$

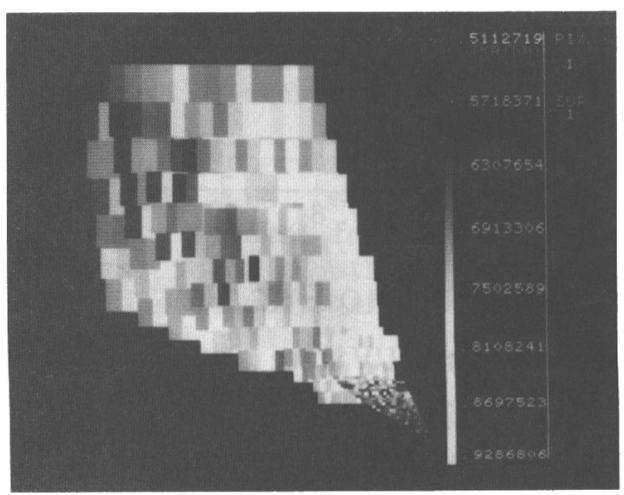

Fig.4 Intensity ratio I 377 / I 482

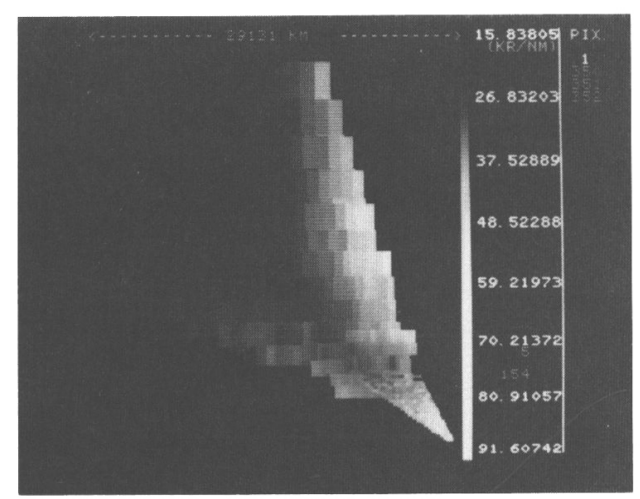

Fig.3 Intensity of $\mathrm{CN}$ emission at $308 \mathrm{~nm}$

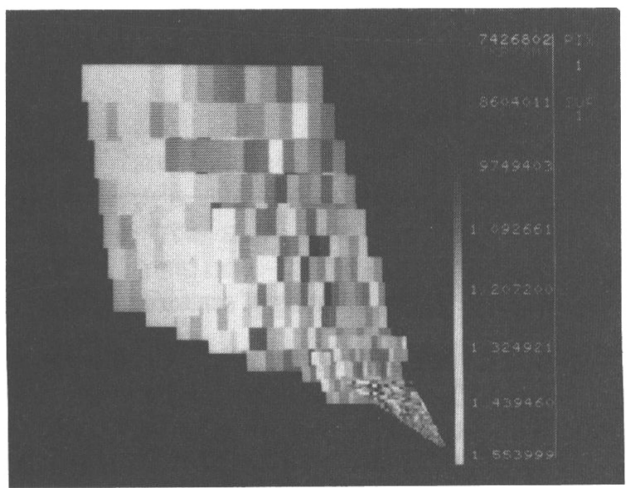

Fig.5 Intensity ratio I 607 / I 482

$377 / 482$ and Qsca 607 / 482. An excess of near-UV radiation is obtained with a particle radius a < $0.4 \mu \mathrm{m}$. An excess of red radiation is obtained with $0.4 \mu \mathrm{m}<\mathrm{a}<0.8 \mu \mathrm{m}$. When the particle radius is $>0.8 \mu \mathrm{m}$, the color effect is less pronounced and disappears when a $>2 \mu \mathrm{m}$. An excess of color in the near-UV and visible range, according to Mie theory, shows that solar radiation is diffracted by tiny submicronic particles. These particles originate partly from the jets which probably constitute a diffuse source (Eberhardt et al., 1987) and partly from the fragmentation of particles of a larger diameter. The fragmentation process very close to the nucleus has been studied in detail by Thomas and Keller (1990). At larger distances, present results suggest that the fragmentation is a noticeable mechanism in the $3200-7000 \mathrm{~km}$ range. The fact that the dust scattered intensity varies as $\mathrm{p}^{-1.52}$ in this region is in good agreement with the Didsy-Hope results of Giotto (Nappo et al., 1989) which mention a local $r^{-s}$ density variation with $s=2.9$ for Didsy and $s=2.6$ for Hope.

\section{Conclusion}

The spatial distribution of dust measured by the solar-scattered continuum shows a $\mathrm{p}^{-\mathrm{s}}$ decrease. The exponent s equals 1, except in a belt approximately located between 3200 and $7000 \mathrm{~km}$ from the nucleus where $s=1.52$. The jets observed in the major molecular emissions are also present in the dust distribution. The color of continuum shows an excess of near-UV radiation between 8000 and $30000 \mathrm{~km}$ correlated with the jets. It shows an excess of red emission at distances higher than 


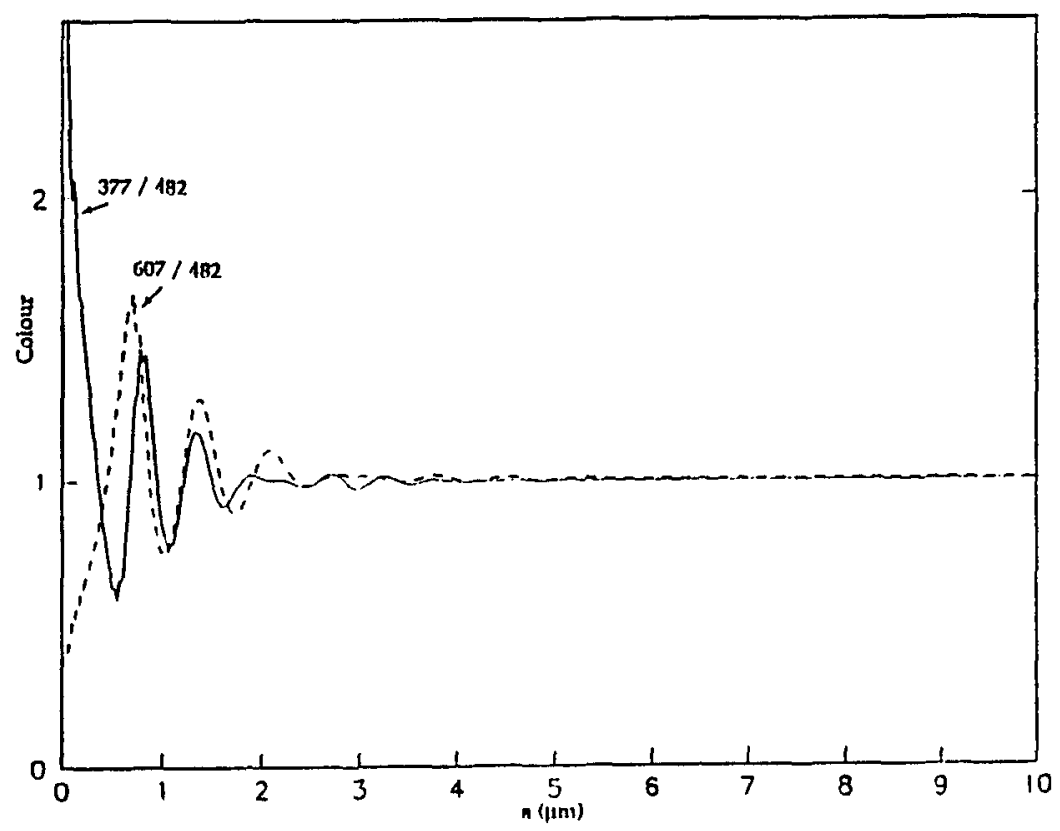

Fig.6 Ratios of scattering efficiencies Qsca 377 / 482 (and Qsca 607 / 482)

$25000 \mathrm{~km}$. A Mie theory calculation shows that an excess of near-UV is due to particles having the radius a $<0.4 \mu \mathrm{m}$ and an excess of red is due to $0.4<\mathrm{a}<0.8 \mu \mathrm{m}$. It is suggested that the submicronic particles responsible for this emission could be produced by fragmentation in the 3200 $7000 \mathrm{~km}$ range and could also originate from the jets which, in this case, constitute a diffuse source.

\section{References}

A'Hearn et al. (1986). Nature 324, 649-651

Clairemidi J., Moreels G., Krasnopolsky V.A., (1990). Icarus, 86, 115-128

Combes et al. (1988). Icarus, 76, 404-436

Eaton N. (1984). Vistas in Astronomy 27, 111-129.

Eberhardt P. et al. (1987). Astron. Astrophys. 187, 481-484

Greenberg J.M., Hage J.I., (1990). Ap. J. $\underline{361}$, 1, 260-274

Hoban S., A'Hearn M.F., Birch P.V., Martin R. (1989). Icarus, 79, 145-158

Jessberger E. K. et al. (1988). Nature 332, 691-695

Keller H. U. et al., (1986). Nature 321, 320-326

Levasseur-Regourd A. C. et al (1986). Nature 321, 341-344

Levasseur-Regourd A. C., Dumont R., Renard J.B., (1990). Icarus, 86, 264-272

Mazets et al. (1987). Astron. Astrophys. 187, 699-706

McDonnell J.A.M. et al. (1987). Astron. Astrophys. 187, 719-741

Moreels et al. (1987). Astron. Astrophys. 187, 551-559

Mukai T., Mukai S., Kikuchi S., (1987). Astron. Astrophys. 187, 650-652

Nappo S. et al. (1989). Asteroids, comets, meteors III, University Uppsala, 397-400

Thomas N., Keller H.U. (1990). Ann. Geophys. 8,2, 147-166

Sagdeev R. Z. et al. (1986). Nature 321, 262-266

Suzuki B., Kurihara H., Watanabe H.,(1990), Publ. Astr. Soc. Japan (Letters), submitted 